\title{
Molecular characterization of clinical isolates from vascular access infection: A single institution study
}

\author{
Yuan-Hsi Tseng ${ }^{1}$, Min Yi Wong ${ }^{1}$, Tsung-Yu Huang ${ }^{1}$, Bor-Shyh Lin² ${ }^{2}$ Chun-Wu Tung ${ }^{1}$, and \\ Yao-Kuang Huang ${ }^{1}$ \\ ${ }^{1}$ Chiayi Chang Gung Memorial Hospital \\ ${ }^{2}$ National Chiao Tung University College of Photonics
}

May 15, 2020

\begin{abstract}
Hemodialysis requires repeated, reliable access to the systemic circulation; therefore, a well-functioning vascular access (VA) procedure is crucial for stable hemodialysis. VA infections (VAIs) constitute the most challenging complication and cause considerable morbidity, loss of access, and even death. In this study, we investigated the molecular profiles of different bacterial isolates retrieved from various types of VA grafts. We collected clinical isolates from hemodialysis patients with VAIs in our institution for the period between 2013 and 2018. We identified the bacterial isolates using standard biochemical procedures; we used a polymerase chain reaction for coagulase-negative staphylococcus (CoNS) and Burkholderia cepacia complex (BCC) species identification. The antibiotic resistance and molecular profile were analyzed using the disk diffusion method and multilocus sequence typing, respectively. We studied 150 isolates retrieved from patients with VAI and observed that Staphylococcus aureus was the predominant bacterial species, followed by S. argenteus, BCC, and CoNS. According to multilocus sequence typing data, we identified a wide variety of sequence types (STs) in S. aureus isolates, with ST59, ST45, and ST239 being the predominant types. Burkholderia cepacia with two new ST types, namely ST1723 and ST1724, accounted for most of the BCC infections, along with ST102 B. contaminans, which were mainly isolated from infected tunneled-cuffed catheters. In summary, the increased incidence of S. argenteus and BCC infections provides insights into their potential clinical effects in VAIs. The various STs identified in different bacterial species indicate the high genetic diversity of bacterial species isolated from VAIs in our institution.
\end{abstract}

\section{Molecular characterization of clinical isolates from vascular access infection: A single institution study}

Yuan-Hsi Tseng*1,2, Min Yi Wong*1,4, Tsung-Yu Huang ${ }^{2,3,4}$, Bor-Shyh Lin ${ }^{5}$, Chun-Wu Tung ${ }^{2,6}$, Yao-Kuang Huang ${ }^{1,2,4+}$

${ }^{1}$ Division of Thoracic and Cardiovascular Surgery, Chiayi Chang Gung Memorial Hospital, Puzi City, Chiayi County 613, Taiwan

${ }^{2}$ College of Medicine, Chang Gung University, Taoyuan City, Taiwan

${ }^{3}$ Division of Infectious Diseases, Department of Internal Medicine, Chiayi Chang Gung Memorial Hospital, Puzi City, Chiayi County 613, Taiwan

${ }^{4}$ Microbiology Research and Treatment Center, Chiayi Chang Gung Memorial Hospital, Puzi City, Chiayi County 613, Taiwan 
${ }^{5}$ Institute of Imaging and Biomedical Photonics, National Chiao Tung University, Tainan 711, Taiwan

${ }^{6}$ Department of Nephrology, Chiayi Chang Gung Memorial Hospital, Puzi City, Chiayi County 613, Taiwan

* Both authors contributed equally to this manuscript

Email addresses of authors:

Yuan-Hsi Tseng: 8802003@cgmh.org.tw

Min Yi Wong: mynyy001@gmail.com

Tsung-Yu Huang: p122273@cgmh.org.tw

Bor-Shyh Lin: borshyhlin@gmail.com

Chun-Wu Tung: Wood5@ms55.hinet.net

+Corresponding author:

Address reprint requests to:

Yao-Kuang Huang, MD, PhD

Division of Thoracic and Cardiovascular Surgery,

Chiayi Chang Gung Memorial Hospital

Address: No. 6, W. Sec., Jiapu Rd., Puzi City, Chiayi County 613, Taiwan (R.O.C.)

Tel: $+886-975368209$

Email: huang137@icloud.com

\begin{abstract}
Hemodialysis requires repeated, reliable access to the systemic circulation; therefore, a well-functioning vascular access (VA) procedure is crucial for stable hemodialysis. VA infections (VAIs) constitute the most challenging complication and cause considerable morbidity, loss of access, and even death. In this study, we investigated the molecular profiles of different bacterial isolates retrieved from various types of VA grafts. We collected clinical isolates from hemodialysis patients with VAIs in our institution for the period between 2013 and 2018. We identified the bacterial isolates using standard biochemical procedures; we used a polymerase chain reaction for coagulase-negative staphylococcus (CoNS) and Burkholderia cepacia complex (BCC) species identification. The antibiotic resistance and molecular profile were analyzed using the disk diffusion method and multilocus sequence typing, respectively. We studied 150 isolates retrieved from patients with VAI and observed thatStaphylococcus aureus was the predominant bacterial species, followed by $S$. argenteus, BCC, and CoNS. According to multilocus sequence typing data, we identified a wide variety of sequence types (STs) in S. aureus isolates, with ST59, ST45, and ST239 being the predominant types. Burkholderia cepacia with two new ST types, namely ST1723 and ST1724, accounted for most of the BCC infections, along with ST102 B. contaminans, which were mainly isolated from infected tunneled-cuffed catheters. In summary, the increased incidence of $S$. argenteus and BCC infections provides insights into their potential clinical effects in VAIs. The various STs identified in different bacterial species indicate the high genetic diversity of bacterial species isolated from VAIs in our institution.
\end{abstract}

\title{
Keywords
}

vascular access infection, multilocus sequence typing,Staphylococcus aureus , Burkholderia cepacia complex, coagulase-negative staphylococcus 


\section{Background}

The population of new patients with end-stage kidney disease (ESKD) receiving dialysis in Taiwan increased from 10,697 in 2013 to 11,596 in 2016. According to the 2018 Annual Report on Kidney Disease in Taiwan, the proportion of new patients with ESKD receiving hemodialysis was $88.9 \%$ in 2013, but it increased to $89.7 \%$ in 2016. The establishment of a well-functioning vascular access (VA) procedure is fundamental to enabling patients to undergo an efficient hemodialysis procedure. Although infection related to VA is not common, it is a problematic complication that may lead to access loss, sepsis, and even death. The major types of VA conduits commonly used are native arteriovenous fistulas (AVFs), prosthetic arteriovenous grafts (AVGs), and central venous catheters (CVCs; both temporary and cuffed tunneled). AVFs and AVGs are preferred over CVCs for dialysis access because CVCs expose patients undergoing hemodialysis to an increased risk of healthcare-associated infections (1). Pathogens primarily responsible for CVC-related infections are Staphylococcus, Gram-negative enteric bacilli, Pseudomonas aeruginosa, and Candida spp. These pathogens can form a biofilm on the CVC walls, rendering them strongly resistant to antibiotic action (2). The mechanism underlying VA infections (VAIs) generally involves the migration of surface organisms along the external surface of the catheter from the exit-site wound or through the lumen of the catheter. Staphylococcus aureus and coagulase-negative staphylococcus (CoNS) species are the most frequently isolated bacteria from VAIs.

S. aureus is among the most common causes of both endemic and epidemic infections acquired in hospitals. Patients undergoing hemodialysis are frequently exposed to $S$. aureus during their stay in dialysis centers, hospitals, or rest homes; the VA site for hemodialysis is a potential site of entry for the pathogen, and the risk of infection is particularly high when a CVC is used $(3,4)$. Furthermore, recent research has reported that CoNS species as the most common etiology of nosocomial bloodstream infection (BSI), especially CVCrelated BSI (CRBSI), in hospitalized patients (5-7).

$P$. aeruginosa is a major cause of nosocomial infection, particularly in immunocompromised patients. It has a predilection for moist environments that serve as its natural reservoirs; therefore, $P$. aeruginosa is a common pathogen in graft infection $(8,9)$.

We conducted a 5-year single-institution study to (1) investigate the prevalence of bacterial species from VAI (2) determine the molecular characteristics of different bacterial species isolated from various types of VAIs, and (3) establish the correlation between bacterial species, sequence types (STs), and VAI types.

\section{Materials and Methods}

\section{Ethical approval}

This study was approved by the Institutional Review Board (IRB) of Chang Gung Memorial Hospital (IRB Numbers: IRB101-41888 and IRB104-8482B). Written consent was obtained from patients, and the study was performed in accordance with approved guidelines.

\section{Study setting and bacterial isolate collection and identification}

This single-institution study was conducted between September 2013 and December 2018 at Chiayi Chang Gung Memorial Hospital, a territory referral hospital in Taiwan. We prospectively collected 150 bacterial isolates from blood and contaminated device samples of 79 patients with VAI who required removal of AVGs and tunneled-cuffed catheters (TCCs). We explained the study procedures to each patient and obtained informed consent prior to performing the procedures. The bacterial isolates were cultured under laboratory standards. The samples were routinely cultured on blood agar at $37{ }^{\circ} \mathrm{C}$ overnight. We performed strain identification through standard biochemical (phenotypic) procedures by using the bioMérieux system.

\section{Antibiotic susceptibility testing}


We subjected all clinical isolates to antimicrobial susceptibility testing against a panel of antimicrobial agents by using the Kirby-Bauer disk diffusion method in accordance with the guidelines of the Clinical and Laboratory Standards Institute (10).

\section{Genomic DNA extraction}

A single colony from a clinical isolate was inoculated in tryptic soy broth (TSB) for $16 \mathrm{~h}$, and $1 \mathrm{~mL}$ of overnight culture was harvested using centrifugation at 16,500 $\times \mathrm{g}$ for $5 \mathrm{~min}$. Bacterial cells were suspended in $1 \mathrm{~mL}$ of ultrapure water and heated at $100{ }^{\circ} \mathrm{C}$ for $15 \mathrm{~min}$. The supernatant containing the DNA was stored at $4{ }^{\circ} \mathrm{C}$ until further use.

\section{Molecular characterization}

\section{CoNS species determination}

To further determine CoNS species, we performed a multiplex polymerase chain reaction (PCR) assay using previously described primer sets $(11,12)$. Ten CoNS species, namely $S$. epidermidis, $S$. haemolyticus , $S$. pasteuri, S. warneri, S. xylosus ,S. capitis, S. caprae, S. saprophyticus, S. lugdunensis, and S. hominis , were determined by the presence and size of the PCR product.

\section{Burkholderia cepacia complex species identification}

We conducted recA sequencing to identify Burkholderia cepacia complex (BCC) species. We performed PCR amplification using specific primers and conditions described by Fehlberg et al. (13). Cycle sequencing was performed using a BigDye Terminator v3.1 cycle sequencing kit and an ABI 3730xl DNA analyzer. We further analyzed the recAsequences and aligned them to a database using NCBI BLASTn.

Detection of mecA and typing of SCCmec for S. aureus and S. epidermidis

To confirm methicillin-resistant $S$. aureus and S. epidermidis, we performed mecA detection using PCR with themecA -specific primer pairs, as described previously (14). We also performed a multiplex PCR assay using four primer pairs to identify SCCmec types I-V (15).

\section{Multilocus sequence typing and phylogenetic analysis}

For the S. aureus, S. epidermidis, P. aeruginosa, and BCC isolates, we conducted multilocus sequence typing (MLST) by amplifying seven housekeeping genes using previously described primer sets (16-19). When aroE of $S$. aureus was not detected, alternative primers were used: aroE745up, 5'-TTATCACCGTCGATGCATAGTGCA-3'; aroE255-down, 5'-CGGAGTAGTATTTATCACAATATC3' (20). Furthermore, we used an alternative forward primer for undetected $\operatorname{trp} B$ of BCC: $\operatorname{trpE}-$ F2, 5'-AAGGACGCGCTGAACGAAGC-3'. The alternative primers used for the undetected tpiA of $S$. epidermidis were as follows: tpi-DF, 5'- GCAAGTATTTGGATAAAAGC-3'; tpi-DR, 5'CCATCTAAGATGATTAAGGC-3'. The allele numbers and STs of each isolate were assigned according to the MLST database (https://pubmlst.org/). We performed advanced cluster analysis to define clonal complexes (CCs) by using BioNumerics software ver. 7.6 (Applied Maths, Sint-Martens-Latem, Belgium).

\section{Typing of spa for $S$. aureus isolates}

For the $S$. aureus and $S$. argenteus isolates, the polymorphic region of the staphylococcal protein A (spa) gene was amplified using previously described primer pairs and sequenced $(21,22)$. We determined spa types using BioNumerics software.

\section{Results}

\section{Analysis of clinical isolates collected from patients with VAIs}


From 2013 to 2018, we collected 150 clinical isolates from patients with VAIs - including AVG- and TCCrelated infections - undergoing hemodialysis in our institution (Figure 1). To investigate the prevalence of different species of bacterial infections across time, we divided the study period into two intervals: (1) from 2013 to 2014 and (2) from 2015 to 2018. The total number of collected isolates decreased in the second interval; however, the prevalence of $S$. aureus infection increased by approximately $20 \%$ (Table 1 ). Moreover, the patients undergoing hemodialysis were mainly infected by Gram-positive bacteria, particularly S. aureus , S. argenteus, and CoNS. P. aeruginosa and BCC species were the main Gram-negative bacteria causing VAIs in our institution.

Regarding species isolation according to VAI types,Staphylococcus spp. were mostly isolated from AVGrelated infections, whereas BCC species were mainly isolated from TCC-related infections.

\section{Molecular characterization of $S$. aureus isolates}

We observed that of $70 \mathrm{~S}$. aureus isolates, 11 were of $S$. argenteus, which is a novel staphylococcal species that is closely related to $S$. aureus genetically and has recently been defined as a part of the $S$. aureus complex (SAC) $(23,24)$. In this study, we identified $S$. argenteus using MLST analysis because the species cannot be distinguished from $S$. aureus through conventional microbiological identification methods. All $S$. argenteus isolates belonged to ST2250 with nontypable spa type, were methicillin susceptible, were mecA negative; however, one isolate carried the SCCmec type I structure.

Among 59 S. aureus isolates, we identified 12 STs. Specifically, ST239, ST45, and ST59 were predominant in methicillin-resistant $S$. aureus (MRSA) isolates, and ST15 and ST7 were predominant in methicillin-sensitive S. aureus (MSSA); ST45, ST59, and ST15 were dominant in blood culture. In addition, ST59, along with ST30 and ST239, was also frequently isolated from contaminated implant devices. ST8, ST15, ST30, and ST45 were more prevalent in AVG isolates than in TCC isolates. Furthermore, we assigned 25 spa types to the isolates, with $\mathrm{t} 437, \mathrm{t} 4864$, t1081, and $\mathrm{t} 091$ being the predominant spatypes. We observed ST8-t008 and ST239-t4864 in both MRSA and MSSA. Moreover, we analyzed the distribution of diverse STs and spatypes among various SCCmec types. ST5-SCCmec IV-t437 (abbreviated as ST5-IV-t437), ST59-V-t437, ST45-V-t081, and ST7-MSSA-t091 were the most prevalent clones in this study.

\section{Molecular characterization of CoNS isolates}

Four staphylococcal species were successfully identified among the 18 CoNS isolates, namely S. epidermidis $(n=9), S$. haemolyticus $(n=2), S$. hominis $(n=1)$, and $S$. lugdunensis $(n=1)$, and five isolates were unclassified; 16 isolates were methicillin resistant (Table 3). Methicillin-resistant S. epidermidis (MRSE) was the predominant species that belonged to seven distinct STs: ST2, ST22, ST57, ST173, ST226, ST490, and ST810. Of the nine MRSE isolates, two carried multiple SCCmec types, and the predominant SCCmec type was type IV. For the $S$. haemolyticus isolates, the oxacillin-susceptible isolate carried mecA and SCCmec type V. Moreover, the identified S. hominis and S. lugdunensis isolates carried SCCmec type II from AVGand TCC-related infections, respectively, and were methicillin resistant. Among the five unidentified CoNS isolates, two were methicillin-resistant CoNS (MR-CoNS) that did not carry mecA. Moreover, of the CoNS isolates, approximately $66.67 \%$ and $33.33 \%$ were isolated from contaminated implant devices and blood culture, respectively. Nevertheless, this study revealed no correlation between ST and origin of isolation.

\section{Molecular characterization of $\boldsymbol{P}$. aeruginosa isolates}

Of nine $P$. aeruginosa isolates, we identified six STs, one of which was a new ST (ST3373). Among the six STs, five were singletons, signifying that they represented only one strain (Table 4). Among the $P$. aeruginosaisolates, nearly $77.8 \%$ were from contaminated implant devices and nearly $22.2 \%$ were from blood culture. We identified a high antibiotic susceptibility rate $(77.78 \% ; 7 / 9)$ for the VAIs, with only two of the nine strains being resistant to antibiotics. ST235, the most prevalent Pseudomonas spp. to have multiple-drug resistance, was resistant to aminoglycoside and fluoroquinolones in this study.

\section{Molecular characterization of BCC isolates}


We identified a total of $13 \mathrm{BCC}$ isolates from TCC-related VAIs; these isolates involved two species, namely B. contaminans and B. cepacia, of which $B$. cepacia was the predominant species (Table 5). MLST typing revealed that B. cepacia strains possessed new MLST types: ST1723 $(n=5)$ and ST1724 $(n=5)$. Most of the isolates that belonged to ST1723 were resistant to imipenem, whereas ST1724 isolates were resistant to gentamicin. Among the BCC isolates, approximately $70 \%$ were from contaminated implant devices and $30 \%$ were from blood culture. However, the study revealed no correlation between the origin of isolation and ST.

\section{Discussion}

VAIs constitute a risk factor for infection in patients undergoing hemodialysis. The pattern of microbes responsible for infection varies substantially among different types of access (25). Pooled data show that $S$. epidermidis accounts for most CVC-related infections, whereas $S$. aureus is more common in AVF- and AVGrelated infections. In our study, staphylococcal species accounted for $58.67 \%$ of VAIs, with S. aureus being the most commonly implicated species, followed by CoNS and S. argenteus. In the 150 isolates collected from patients with VAIs, $S$. aureus was the predominant pathogen in AVG- and TCC-related infections, with a rate of $37 / 79(46.84 \%)$ and $22 / 71(30.99 \%)$, respectively. S. argenteus, another in SAC species, was also more predominant in AVG-related infections than in TCC-related infections. Notably, the nine $S$. epidermidis isolates were mainly collected from AVG-related infections (6/9); this finding is not consistent with those reported by a previous study (26), which indicated that improving sterilization management procedures during hemodialysis may reduce the number of skin clones such as S. epidermidis on TCCs. Regarding representative Gram-negative bacteria in VAIs, $P$. aeruginosa and BCC predominantly caused TCC infections; in particular, BCC caused only TCC infections.

In patients undergoing hemodialysis, $S$. aureus infection is common, especially MRSA infection, the incidence of which was reported to be higher than that observed in the general population by 100-fold (27). In our study, MRSA and MSSA infections accounted for $62.71 \%$ and $37.29 \%$ of $S$. aureus VAIs, respectively, with ST45, ST59, and ST239 being the predominant clones. Compared with our previous study,(4) the present study revealed that ST45, ST59, and ST239 were common in other diseases or surgical infections, indicating that these are major clones in our institution and warrant more attention. Notably, we also found the $S$. aureus ST239 - an emerging multidrug-resistant MRSA clone worldwide that generally carries an SCCmec type III element - in methicillin-sensitive strains without mecA . Furthermore, a novel nonpigmented staphylococcal lineage that cannot be distinguished from $S$. aureus using routine microbiological identification methods is now formally classified as $S$. argenteus ; it was initially described as part of the distinct $S$. aureus CC (CC75) that is prevalent in aboriginal communities in the Northern Territory of Australia (28). S. argenteus comprising several CCs with many STs, especially ST2250, is the most commonly reported lineage with an extensive geographic distribution, including France, Belgium, Thailand, Taiwan, Japan, and China, indicating a global spread (29-35). The widespread $S$. argenteus has been isolated from both humans and animals. In our institution, ST2250 was the primary and only methicillin-sensitive ST revealed in VAIs, a finding that is consistent with those for previously reported $S$. argenteus -infected bacteremia cases in Taiwan (33).

The BCC is a group of opportunistic pathogens comprising at least 20 different species that commonly cause infections in immunocompromised patients, particularly those with cystic fibrosis $(\mathrm{CF})$. B. contaminans was first identified from a contaminated Sargasso Sea DNA sample (36) and is increasingly associated with CF. However, other hospitalized non-CF patients have been reported to be affected by $B$. contaminans and $B$. cepacia infections. Nevertheless, $B$. contaminans has been found to be a contaminant in manufactured products, including pharmaceuticals and disinfectants $(37,38)$. In our institution, we obtained all BCC isolates from infected TCCs in hemodialysis patients with VAI; this suggests that the repeated use of mechanical device detergent and hemodialyzer reprocessing may cause contamination and that BCC species can survive in a harsh environment.

In this 5-year study, we collected 150 isolates from hemodialysis patients with VAIs and analyzed the isolates on the basis of the year of isolation (i.e., study period interval). Although the number of isolates from infected 
accesses was relatively low in the interval 2015-2018, the incidence of $S$. aureus , S. argenteus , and BCC infections increased by approximately $10 \%$ (i.e., $45.76 \%, 13.56 \%$, and $13.56 \%$, respectively). By contrast, CoNS and $P$. aeruginosainfections decreased by nearly 3\%-5\%. Previous studies have not addressed the spread or transmission of $S$. argenteus in the hospital environment (39). Nevertheless, the growing trend of $S$. argenteus in VAIs indicates the potential and importance of this novel, difficult-to-delimit species in healthcare-associated infections. Therefore, infection prevention and control measures that can be applied for $S$. aureus can be adopted for S. argenteus .

\section{Study limitations}

The major limitation of this study is that the examined VAIs were mainly responsible for the removal of access. By contrast, we did not include infections managed through early intervention with conservative antibiotic treatment after identification. Therefore, we could not provide an overview of VAIs in this study.

\section{Conclusions}

In this study, we examined 150 clinical isolates retrieved from infected VA grafts, including AVGs and TCCs, in hemodialysis patients by conducting a 5-year epidemiological surveillance at a single institution in Taiwan. The three major STs (i.e., ST239, ST59, and ST45) of MRSA with various spa types showed high genetic diversity in S. aureus VAIs. Moreover, the ST102 B. contaminans isolate and two newly identified STs, namely ST1723 and ST1724 B. cepaciaisolates, were exclusively retrieved from TCC-related infections. The increased incidence of infections engendered by $S$. argenteus and BCC provides insight into the potential clinical effects of $S$. argenteus and BCC species in VAIs.

\section{Acknowledgment}

We thank Wallace Academic Editing for editing this manuscript. This study was supported by grants from Chang Gung Memorial Hospital, Chiayi, Taiwan (Grant numbers: CMRPG6J0401, CMRPG6J0402, CMRPG6J0403, CMRPG6H0292, and CMRPG6H0293).

\section{Conflict of Interests}

The authors have no conflict of interest to declare.

\section{Author Contributions}

Yuan-Hsi Tseng: Conceptualization (lead); methodology (lead); funding acquisition (lead); writing - original draft (lead); writing - review and editing (equal); Min Yi Wong: Conceptualization (lead); investigation (lead); writing - original draft (lead); formal analysis (lead); writing - review and editing (equal); Tsung-Yu Huang, Bor-Shyh Lin, Chun-Wu Tung: Investigation (supporting); formal analysis (supporting); writing - original draft (supporting); writing - review and editing (equal); Yao-Kuang Huang: Conceptualization (supporting); funding acquisition (lead); writing - original draft (supporting); writing - review and editing (equal). All authors read and approved the final manuscript.

\section{Ethics Statement}

This study was approved by the Institutional Review Board (IRB) of Chang Gung Memorial Hospital (IRB Nos: IRB101-41888 and IRB104-8482B). 


\section{Data Accessibility Statement}

The authors declare that the experimental data published in this paper are made accessible upon request for interested readers.

\section{References}

1. Lafrance JP, Rahme E, Lelorier J, Iqbal S. Vascular access-related infections: definitions, incidence rates, and risk factors. Am J Kidney Dis. 2008;52(5):982-93.

2. Santoro D, Benedetto F, Mondello P, Pipito N, Barilla D, Spinelli F, et al. Vascular access for hemodialysis: current perspectives. Int J Nephrol Renovasc Dis. 2014;7:281-94.

3. Scheuch M, Freiin von Rheinbaben S, Kabisch A, Engesser J, Ahrendt S, Dabers T, et al. Staphylococcus aureus colonization in hemodialysis patients: a prospective 25 months observational study. BMC Nephrol. 2019;20(1):153.

4. Chu C, Wong MY, Tseng YH, Lin CL, Tung CW, Kao CC, et al. Vascular access infection by Staphylococcus aureus from removed dialysis accesses. Microbiologyopen. 2019:e800.

5. Freixas N, Bella F, Limon E, Pujol M, Almirante B, Gudiol F. Impact of a multimodal intervention to reduce bloodstream infections related to vascular catheters in non-ICU wards: a multicentre study. Clin Microbiol Infect. 2013;19(9):838-44.

6. Hebeisen UP, Atkinson A, Marschall J, Buetti N. Catheter-related bloodstream infections with coagulasenegative staphylococci: are antibiotics necessary if the catheter is removed? Antimicrob Resist Infect Control. 2019;8:21.

7. Lebeaux D, Fernández-Hidalgo N, Chauhan A, Lee S, Ghigo J-M, Almirante B, et al. Management of infections related to totally implantable venous-access ports: challenges and perspectives. Lancet Infect Dis. 2014;14(2):146-59.

8. Chen YK, Fang HC, Chou KJ, Lee PT, Chung HM. A puzzling cause of persistent Pseudomonas aeruginosa septicaemia in a patient on maintenance haemodialysis. Nephrol Dial Transplant. 2004;19(9):2400-2.

9. Pham TM, Kretzschmar M, Bertrand X, Bootsma M, Consortium C-M. Tracking Pseudomonas aeruginosa transmissions due to environmental contamination after discharge in ICUs using mathematical models. PLoS Comput Biol. 2019;15(8):e1006697.

10. Institute CLS. Performance standards for antimicrobial susceptibility testing; Twenty-third international supplement. CLSI document M100-S23. CLSI Wayne, PA2013.

11. Kim J, Hong J, Lim JA, Heu S, Roh E. Improved multiplex PCR primers for rapid identification of coagulase-negative staphylococci. Arch Microbiol. 2018;200(1):73-83.

12. Campos-Pena E, Martin-Nunez E, Pulido-Reyes G, Martin-Padron J, Caro-Carrillo E, Donate-Correa J, et al. Multiplex PCR assay for identification of six different Staphylococcus spp. and simultaneous detection of methicillin and mupirocin resistance. J Clin Microbiol. 2014;52(7):2698-701.

13. Fehlberg LC, Andrade LH, Assis DM, Pereira RH, Gales AC, Marques EA. Performance of MALDI-ToF MS for species identification ofBurkholderia cepacia complex clinical isolates. Diagn Microbiol Infect Dis. 2013;77(2):126-8.

14. Pournajaf A, Ardebili A, Goudarzi L, Khodabandeh M, Narimani T, Abbaszadeh H. PCR-based identification of methicillin-resistantStaphylococcus aureus strains and their antibiotic resistance profiles. Asian Pac J Trop Biomed. 2014;4(Suppl 1):S293-7. 
15. Boye K, Bartels MD, Andersen IS, Moller JA, Westh H. A new multiplex PCR for easy screening of methicillin-resistant Staphylococcus aureus SCCmec types I-V. Clin Microbiol Infect. 2007;13(7):725-7.

16. Enright MC, Day NP, Davies CE, Peacock SJ, Spratt BG. Multilocus sequence typing for characterization of methicillin-resistant and methicillin-susceptible clones of Staphylococcus aureus . J Clin Microbiol. 2000;38(3):1008-15.

17. Curran B, Jonas D, Grundmann H, Pitt T, Dowson CG. Development of a multilocus sequence typing scheme for the opportunistic pathogenPseudomonas aeruginosa. J Clin Microbiol. 2004;42(12):5644-9.

18. Spilker T, Baldwin A, Bumford A, Dowson CG, Mahenthiralingam E, LiPuma JJ. Expanded multilocus sequence typing for Burkholderiaspecies. J Clin Microbiol. 2009;47(8):2607-10.

19. Thomas JC, Vargas MR, Miragaia M, Peacock SJ, Archer GL, Enright MC. Improved multilocus sequence typing scheme for Staphylococcus epidermidis . J Clin Microbiol. 2007;45(2):616-9.

20. Ruimy R, Armand-Lefevre L, Barbier F, Ruppe E, Cocojaru R, Mesli Y, et al. Comparisons between geographically diverse samples of carriedStaphylococcus aureus. J Bacteriol. 2009;191(18):5577-83.

21. Strommenger B, Braulke C, Heuck D, Schmidt C, Pasemann B, Nubel U, et al. spa Typing of Staphylococcus aureus as a frontline tool in epidemiological typing. J Clin Microbiol. 2008;46(2):574-81.

22. Schuster D, Rickmeyer J, Gajdiss M, Thye T, Lorenzen S, Reif M, et al. Differentiation of Staphylococcus argenteus (formerly:Staphylococcus aureus clonal complex 75) by mass spectrometry from $S$. aureus using the first strain isolated from a wild African great ape. Int J Med Microbiol. 2017;307(1):57-63.

23. Aung MS, San T, San N, Oo WM, Ko PM, Thet KT, et al. Molecular characterization of Staphylococcus argenteus in Myanmar: identification of novel genotypes/clusters in staphylocoagulase, protein A, alphahaemolysin and other virulence factors. J Med Microbiol. 2019;68(1):95-104.

24. Jiang B, You B, Tan L, Yu S, Li H, Bai G, et al. ClinicalStaphylococcus argenteus Develops to Small Colony Variants to Promote Persistent Infection. Front Microbiol. 2018;9:1347.

25. Tokars JI, Miller ER, Stein G. New national surveillance system for hemodialysis-associated infections: initial results. Am J Infect Control. 2002;30(5):288-95.

26. Saeed Abdulrahman I, Al-Mueilo SH, Bokhary HA, Ladipo GO, Al-Rubaish A. A prospective study of hemodialysis access-related bacterial infections. J Infect Chemother. 2002;8(3):242-6.

27. Control CfD, Prevention. Invasive methicillin-resistantStaphylococcus aureus infections among dialysis patients-United States, 2005. MMWR Morbidity and mortality weekly report. 2007;56(9):197.

28. Ng JW, Holt DC, Lilliebridge RA, Stephens AJ, Huygens F, Tong SY, et al. Phylogenetically distinct Staphylococcus aureus lineage prevalent among indigenous communities in northern Australia. J Clin Microbiol. 2009;47(7):2295-300.

29. Dupieux C, Blonde R, Bouchiat C, Meugnier H, Bes M, Laurent S, et al. Community-acquired infections due to Staphylococcus argenteuslineage isolates harbouring the Panton-Valentine leucocidin, France, 2014. Eurosurveillance. 2015;20(23):21154.

30. Argudin MA, Dodemont M, Vandendriessche S, Rottiers S, Tribes C, Roisin S, et al. Low occurrence of the new species Staphylococcus argenteus in a Staphylococcus aureus collection of human isolates from Belgium. Eur J Clin Microbiol Infect Dis. 2016;35(6):1017-22.

31. Chantratita N, Wikraiphat C, Tandhavanant S, Wongsuvan G, Ariyaprasert P, Suntornsut P, et al. Comparison of community-onsetStaphylococcus argenteus and Staphylococcus aureus sepsis in Thailand: a prospective multicentre observational study. Clin Microbiol Infect. 2016;22(5):458 e11-9. 
32. Moradigaravand D, Jamrozy D, Mostowy R, Anderson A, Nickerson EK, Thaipadungpanit J, et al. Evolution of the Staphylococcus argenteus ST2250 Clone in Northeastern Thailand Is Linked with the Acquisition of Livestock-Associated Staphylococcal Genes. mBio. 2017;8(4).

33. Chen SY, Lee H, Wang XM, Lee TF, Liao CH, Teng LJ, et al. High mortality impact of Staphylococcus argenteus on patients with community-onset staphylococcal bacteraemia. Int J Antimicrob Agents. 2018;52(6):747-53.

34. Ohnishi T, Shinjoh M, Ohara H, Kawai T, Kamimaki I, Mizushima R, et al. Purulent lymphadenitis caused by Staphylococcus argenteus, representing the first Japanese case of Staphylococcus argenteus (multilocus sequence type 2250) infection in a 12-year-old boy. J Infect Chemother. 2018;24(11):925-7.

35. Li Q, Li Y, Tang Y, Meng C, Ingmer H, Jiao X. Prevalence and characterization of Staphylococcus aureus andStaphylococcus argenteus in chicken from retail markets in China. Food Control. 2019;96:158-64.

36. Mahenthiralingam E, Baldwin A, Drevinek P, Vanlaere E, Vandamme P, LiPuma JJ, et al. Multilocus sequence typing breathes life into a microbial metagenome. PLoS One. 2006;1:e17.

37. Martin M, Christiansen B, Caspari G, Hogardt M, von Thomsen AJ, Ott E, et al. Hospital-wide outbreak of Burkholderia contaminanscaused by prefabricated moist washcloths. J Hosp Infect. 2011;77(3):267-70.

38. Moehring RW, Lewis SS, Isaacs PJ, Schell WA, Thomann WR, Althaus MM, et al. Outbreak of bacteremia due to Burkholderia contaminanslinked to intravenous fentanyl from an institutional compounding pharmacy. JAMA Intern Med. 2014;174(4):606-12.

39. Becker K, Schaumburg F, Kearns A, Larsen AR, Lindsay JA, Skov RL, et al. Implications of identifying the recently defined members of theStaphylococcus aureus complex S. argenteus and S. schweitzeri : a position paper of members of the ESCMID Study Group for Staphylococci and Staphylococcal Diseases (ESGS). Clin Microbiol Infect. 2019;25(9):1064-70.

Tables

Table 1. Prevalence (\%) of 150 isolated vascular access infection (VAI) pathogens among hemodialysis patients in Chang Gung Memorial Hospital in Chiayi

\begin{tabular}{|c|c|c|c|c|c|c|c|}
\hline & $\begin{array}{l}\text { Bacterial } \\
\text { isolates } \\
\text { (Total } \\
\mathbf{n}=150 \text { ) }\end{array}$ & $\begin{array}{l}\text { Bacterial } \\
\text { isolates } \\
\text { (Total } \\
\mathbf{n}=150 \text { ) }\end{array}$ & $\begin{array}{l}\text { Bacterial } \\
\text { isolates } \\
\text { (Total } \\
\mathbf{n}=150 \text { ) }\end{array}$ & $\begin{array}{l}\text { Bacterial } \\
\text { isolates } \\
\text { (Total } \\
\mathbf{n}=150 \text { ) }\end{array}$ & $\begin{array}{l}\text { Bacterial } \\
\text { isolates } \\
\text { (Total } \\
\mathbf{n}=150 \text { ) }\end{array}$ & $\begin{array}{l}\text { Bacterial } \\
\text { isolates } \\
\text { (Total } \\
\mathbf{n}=150 \text { ) }\end{array}$ & Total \\
\hline & $\begin{array}{l}S . \text { aureus } S \text {. } \\
\text { argenteus }\end{array}$ & CoNS & $\begin{array}{l}P . \\
\text { aeruginosa }\end{array}$ & $\mathrm{BCC}$ & Others & Others & \\
\hline No. & $\begin{array}{l}70 \\
(46.67 \%)\end{array}$ & $18(12 \%)$ & $10(6.67 \%)$ & $13(8.67 \%)$ & $\begin{array}{l}\mathrm{G}(+)^{\mathrm{a}} \\
17 \\
(11.33 \%)\end{array}$ & $\begin{array}{l}\mathrm{G}(-) \mathrm{b} \\
22 \\
(14.67 \%)\end{array}$ & 150 \\
\hline \multicolumn{8}{|l|}{ Year } \\
\hline 2013-2014 & $\begin{array}{l}35 \\
(38.46 \%)\end{array}$ & $\begin{array}{l}12 \\
(13.19 \%)\end{array}$ & $8(8.79 \%)$ & $5(5.49 \%)$ & $\begin{array}{l}14 \\
(15.38 \%)\end{array}$ & $\begin{array}{l}17 \\
(18.68 \%)\end{array}$ & 91 \\
\hline 2015-2018 & $\begin{array}{l}35 \\
(59.32 \%)\end{array}$ & $6(10.17 \%)$ & $2(3.39 \%)$ & $8(13.56 \%)$ & $3(5.08 \%)$ & $5(8.47 \%)$ & 59 \\
\hline \multicolumn{8}{|l|}{ Origin } \\
\hline AVG & 45 & 10 & 4 & 0 & 9 & 11 & 79 \\
\hline \multicolumn{8}{|l|}{ Isolation } \\
\hline Blood & 17 & 6 & 2 & 4 & 3 & 3 & 35 \\
\hline Others ${ }^{c}$ & 53 & 12 & 8 & 9 & 14 & 19 & 115 \\
\hline
\end{tabular}


a. Others G (+) included Corynebacterium spp.,Corynebacterium jeikeium, Clostridium perfringens ,Enterococcus faecalis, Enterococcus faecium, Group DStreptococcus (GDS), Streptococcus agalactiae,

Viridans streptococcus

b. Others G (-) included Acinetobacter baumannii , Bacteroides fragilis ,Bacteroides thetaiotaomicron, Citrobacter koseri ,Escherichia coli, Escherichia spp., Enterobacter cloacae, Klebsiella oxytoca, Klebsiella pneumoniae ,Morganella morganii, Proteus mirabilis, Proteus vulgaris, Stenotrophomonas maltophilia

c. Others included abscess, AV-shunt, body fluid, CVP, graft, Hickman, pus, tissue, and wound.

Table 2. Distribution of MLST, spa types, and SCCmec types in different isolates of MRSA and MSSA clones according to MLST clone complex (CC).

\begin{tabular}{|c|c|c|c|c|c|c|}
\hline & $\mathrm{CC}^{\mathrm{a}}$ & ST type & spa type & $\mathrm{SCC} m e c$ & Case & Isolation \\
\hline \multirow[t]{8}{*}{ MRSA $\mathrm{n}=37$} & CC5 & 5 & t002(2) & II (2) & $\begin{array}{l}\text { AVG (1), TCC } \\
\text { (1) }\end{array}$ & $\begin{array}{l}\text { Blood (1), } \\
\text { Others (1) }\end{array}$ \\
\hline & $\mathrm{CC} 8$ & 8 & t008(1) & IV (1) & AVG (1) & Others (1) \\
\hline & & 239 & $\begin{array}{l}\text { t4864(2), } \\
\text { t3528(1), } \\
\text { t037(2), } \\
\text { t748(1) }\end{array}$ & II (2), III (4) & $\begin{array}{l}\text { AVG (2), } \\
\text { TCC (4) }\end{array}$ & $\begin{array}{l}\text { Blood (1), } \\
\text { Others (5) }\end{array}$ \\
\hline & & 4798 & t037(1) & III (1) & $\mathrm{TCC}(1)$ & Others (1) \\
\hline & CC30 & 30 & $\begin{array}{l}\text { t019(4), } \\
\text { t1836(1) }\end{array}$ & IV (5) & $\begin{array}{l}\text { AVG (3), } \\
\text { TCC (2) }\end{array}$ & Others (5) \\
\hline & CC45 & 45 & $\begin{array}{l}\text { t002(1), } \\
\text { t026(3), } \\
\text { t1081(4), } \\
\text { t2383(1) }\end{array}$ & $\begin{array}{l}\text { II (1), IV } \\
(4), \text { V (4) }\end{array}$ & $\begin{array}{l}\text { AVG (6), } \\
\text { TCC (3) }\end{array}$ & $\begin{array}{l}\text { Blood (4), } \\
\text { Others (5) }\end{array}$ \\
\hline & & 508 & t026(1) & $\mathrm{NT}(1)$ & AVG (1) & Others (1) \\
\hline & Other & 59 & $\begin{array}{l}\text { t437(7), } \\
\text { t3513(3), } \\
\text { t3527(2) }\end{array}$ & $\mathrm{IV}(7), \mathrm{V}(5)$ & $\begin{array}{l}\text { AVG (6), } \\
\text { TCC (6) }\end{array}$ & $\begin{array}{l}\text { Blood (3), } \\
\text { Others (9) }\end{array}$ \\
\hline \multirow{10}{*}{ MSSA $n=22$} & $\mathrm{CC} 1$ & 1 & $\mathrm{t} 2457(1)$ & NA (1) & TCC (1) & Others (1) \\
\hline & & 188 & $\begin{array}{l}\text { t2769(1), } \\
\text { t189(1) }\end{array}$ & $\mathrm{I}(1), \mathrm{NA}(1)$ & $\begin{array}{l}\operatorname{AVG}(1), \\
\operatorname{TCC}(1)\end{array}$ & Others (2) \\
\hline & $\mathrm{CC} 8$ & 8 & t008(2) & NA (2) & AVG (2) & Others (2) \\
\hline & & 239 & $\mathrm{t} 4864(2)$ & NA (2) & AVG (2) & Others (2) \\
\hline & $\mathrm{CC} 15$ & 15 & $\begin{array}{l}\text { t803(2), } \\
\text { t279(2), } \\
\text { t547(1), } \\
\text { t084(1) }\end{array}$ & NA (6) & $\begin{array}{l}\text { AVG (5), } \\
\text { TCC (1) }\end{array}$ & $\begin{array}{l}\text { Blood (3), } \\
\text { Others (3) }\end{array}$ \\
\hline & CC30 & 30 & $\mathrm{t} 3732(1)$ & NA (1) & AVG (1) & Others (1) \\
\hline & CC97 & 97 & $\mathrm{t} 224(1)$ & NA (1) & AVG (1) & Others (1) \\
\hline & Other & 7 & $\mathrm{t} 091(4)$ & NA (4) & $\begin{array}{l}\text { AVG }(2), \\
\text { TCC (2) }\end{array}$ & $\begin{array}{l}\text { Blood (1), } \\
\text { Others (3) }\end{array}$ \\
\hline & & 398 & $\mathrm{t} 571(1)$ & NA (1) & AVG (1) & Others (1) \\
\hline & & 845 & $\mathrm{t} 084(2)$ & NA (2) & AVG (2) & $\begin{array}{l}\text { Blood (1), } \\
\text { Others (1) }\end{array}$ \\
\hline
\end{tabular}

${ }^{\mathrm{a}} \mathrm{CC}$ : clonal complex

AVG: arteriovenous graft; TCC: tunneled-cuffed catheter 
NT: nontypeable, no corresponding band was found in multiplex PCR for SCCmec typing.

NA: not applicable

Table 3. Molecular characterization of methicillin-resistant and methicillin-susceptible coagulase-negative Staphylococcus (CoNS) isolates from vascular access infections.

\begin{tabular}{|c|c|c|c|c|c|c|}
\hline Species & & ST type & Case & Isolation & $\mathrm{SCC} m e c$ & No. \\
\hline \multirow{8}{*}{$\begin{array}{l}\text { S. epidermidis } \\
\mathrm{n}=9\end{array}$} & MRSE & 2 & TCC & Others & IV & 1 \\
\hline & & 22 & AVG & Others & I & 1 \\
\hline & & & TCC & Blood & & 1 \\
\hline & & 57 & TCC & Others & IV & 1 \\
\hline & & 173 & AVG & Others & $\mathrm{IV}+\mathrm{V}$ & 1 \\
\hline & & 226 & AVG & Others & IV & 1 \\
\hline & & 490 & AVG & Others & $\mathrm{I}+\mathrm{III}$ & 2 \\
\hline & & 810 & AVG & Blood & IV & 1 \\
\hline \multirow{2}{*}{$\begin{array}{l}\text { S. } \\
\text { haemolyticus } \\
\mathrm{n}=2\end{array}$} & MRSH & 1 & TCC & Blood & V & 1 \\
\hline & MSSH & 9 & AVG & Others & $\mathrm{V}$ & 1 \\
\hline $\begin{array}{l}\text { S. hominis } \\
\mathrm{n}=1\end{array}$ & MRSHo & ND & AVG & Blood & $\mathrm{NT}$ & 1 \\
\hline $\begin{array}{l}\text { S. lugdunensis } \\
\mathrm{n}=1\end{array}$ & MRSL & ND & TCC & Others & II & 1 \\
\hline \multirow{4}{*}{$\begin{array}{l}\text { Coag(-) } \\
\text { Staphylococcus } \\
\mathrm{n}=5\end{array}$} & MR-CoNS & ND & TCC & Blood & NT & 2 \\
\hline & & ND & TCC & Others & $\mathrm{NA}$ & 1 \\
\hline & & ND & AVG & Others & $\mathrm{NA}$ & 1 \\
\hline & MS-CoNS & ND & AVG & Others & $\mathrm{NA}$ & 1 \\
\hline
\end{tabular}

AVG: arteriovenous graft; TCC: tunneled-cuffed catheter

ND: not determined

NA: not applicable

NT: non-typeable, no corresponding band was found in multiplex PCR for SCCmec typing.

Table 4. Distribution of MLST and antibiotic resistance of P. aeruginosa isolated from different types of access.

\begin{tabular}{lllll}
\hline ST type & Case & Isolation & Antibiotic resistance profile & No. \\
\hline 235 & TCC & Blood & CIP, GEN, LVX & 1 \\
244 & AVG & Blood & NONE & 1 \\
& TCC & Others & & 1 \\
303 & AVG & Others & CAZ, PIP, TZP & 1 \\
381 & TCC & Others & NONE & 1 \\
2682 & AVG & Others & & 1 \\
3373 & TCC & Others & & 2 \\
ND & TCC & Others & & 1
\end{tabular}




\begin{tabular}{lllll}
\hline ST type & Case & Isolation & Antibiotic resistance profile & No. \\
\hline Total & Total & Total & Total & 9 \\
\hline
\end{tabular}

AVG: arteriovenous graft; TCC: tunneled-cuffed catheter

CIP: ciprofloxacin; GEN: gentamicin; LVX: levofloxacin; CAZ: ceftazidime; PIP: piperacillin

Table 5. Distribution of MLST and antibiotic resistance of B. cepacia complex (BCC) isolated from different types of vascular access.

\begin{tabular}{|c|c|c|c|c|c|}
\hline Species & ST type & Case & Isolation & $\begin{array}{l}\text { Antibiotic } \\
\text { resistance } \\
\text { profile }\end{array}$ & No. \\
\hline \multirow[t]{2}{*}{$\begin{array}{l}\text { B. contaminans } \\
\mathrm{n}=3\end{array}$} & 102 & TCC & Others & CST & 2 \\
\hline & & & Blood & CST & 1 \\
\hline \multirow[t]{9}{*}{ B. cepacia $\mathrm{n}=10$} & 1723 & TCC & Others & CST, GEN, IPM & 2 \\
\hline & & & & CST, IPM & 1 \\
\hline & & & & IPM, DOR & 1 \\
\hline & & & Blood & $\mathrm{ND}$ & 1 \\
\hline & 1724 & & Blood & $\begin{array}{l}\text { CST, GEN, } \\
\text { IPM }\end{array}$ & 1 \\
\hline & & & Others & $\begin{array}{l}\text { CST, GEN, } \\
\text { IPM }\end{array}$ & 1 \\
\hline & & & Blood & GEN & 1 \\
\hline & & & Others & GEN & 1 \\
\hline & & & Others & No & 1 \\
\hline Total & Total & Total & Total & Total & 13 \\
\hline
\end{tabular}

TCC: tunneled-cuffed catheter

CST: colistin; GEN: gentamicin; IPM: imipenem: DOR: doripenem

Figure Legend

Figure 1. Distribution of isolates from vascular access infections in hemodialysis patients. 
Distribution of Bacterial Isolate from Hemodialysis Patients

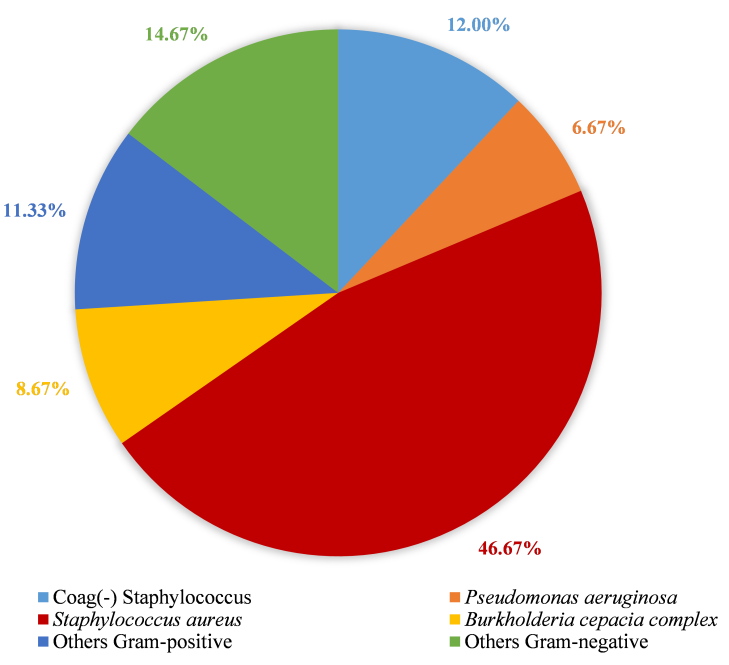

